$\$=1$

\title{
Post menopause and Osteoporosis - Unfolding the link
}

\author{
Dathar Sahithi ${ }^{1 *}$, Sudhakara Reddy ${ }^{2}$, N.Rajesh ${ }^{1}$, Sheela Priyadarshini ${ }^{3}$, \\ Meenakshi Krishnan ${ }^{3}$, Gurugubelli Upendra ${ }^{1}$ \\ ${ }^{1}$ Post graduate student, Department of Oral medicine and Radiology, Vishnu Dental College \\ ${ }^{2}$ Professor and Head of Department of Oral medicine and Radiology, Vishnu Dental College \\ ${ }^{3}$ Assistant Professor of Department of Oral medicine and Radiology, Vishnu Dental College \\ *Corresponding author E-mail: sahithi.dathar9@gmail.com
}

\begin{abstract}
Background: Osteoporosis is one of the most systemic diseases prevalent among the postmenopausal women attributing to the increased risk of occurrence of fractures. Hence, early detection of this disease is of utmost importance which can be determined by concentration of calcium in saliva and radiographically by quantitative radiomorphometric indices like panoramic mandibular Index (PMI).

Objectives: The purpose of this study is to evaluate and compare the salivary calcium levels and PMI in order to identify the risk group of osteoporosis in postmenopausal women.

Methods: A case-control study was carried out among 60 female subjects who were equally categorized into two groups. Salivary calcium levels were calorimetrically assessed by spectrophotometry and PMI index was calculated using Digora software from the Digital Panoramic image obtained for individual patient.

Results: The results had shown that salivary calcium among the case group was significantly higher than the control group ( $\mathrm{p}$ value $=$ 0.001 ) and PMI values among the case group were significantly lower than the control group ( $\mathrm{p}$ value $=0.001$ ). With the advancing duration of menopause, the salivary calcium levels were increasing and PMI values were found to be decreasing and both were statistically significant.

Conclusion: The results of this study suggest that both salivary calcium and PMI can certainly be used as screening tools to identify the risk of osteoporosis among postmenopausal women.
\end{abstract}

Keywords: Osteoporosis; Post-Menopausal Women; Panoramic Mandibular Index; Radiomorphometric Indices; Salivary Calcium.

\section{Introduction}

Osteoporosis, a silent disease, is perhaps the most common regressive disease of the bone, frequently witnessed among the postmenopausal women. Osteoporosis is a chronic systemic skeletal disease characterized by a decreased bone mass and disrupted bone tissue microstructures, resulting in increased bone fragility and increased risk of fracture (Poornima et al. 2014). The major obstacle of this disease is that it is usually not discovered until weakened bones cause pain and debilitating fractures (Haster et al. 2011).

Menopause being a natural physiological process occurs during fourth to fifth decade in a life of a woman and tends to increase a woman's susceptibility to osteoporosis (Duncea et al.2013).

There has often been a greater intricacy in identifying this combating disease before the clinical consequences have occurred Though there are different strategies for screening of osteoporosis, Central dual-energy X-ray (DXA) is known to be the gold standard (Haster et al. 2011). For the past few decades, there is a vast growing importance in identifying the risk of osteoporosis by noninvasive and less expensive investigations so as to reach the hands of a common man.

Saliva being the mirror of systemic diseases, its role has advanced elegantly and has steadily gained importance in various diagnostic and research arenas (Siva Reddy et al. 2008). Calcium is a key nutrient and an essential building block imparting a lifelong bone health. It is an abundant mineral in almost every cell of the human body.

Calcium accounts as the main mineral component of the human skeletal system. Resorption of bone thus can result in diffusion of calcium into blood and further into the saliva (Kavitha et al. 2012). Increased levels of calcium in saliva can thus be used as a potential screening tool for assessing an individual risk for osteoporosis.

According to Maryam Rabiei.et al the cut-off point for salivary calcium is $6.1 \mathrm{mg} / \mathrm{dl}$ and above which (i.e. $>6.1 \mathrm{mg} / \mathrm{dl}$ ) can used as a screening tool to identify risk group of osteoporosis in postmenopausal women. (Maryam Rabiei et al. 2012).

Radiographic imaging is an important diagnostic adjunct and has heralded a major progress in providing a comprehensive image of jaws and maxillofacial structures. Dental Panoramic radiographs are being used as auxiliary tools for assessing the mandibular cortical width among osteoporosis patients (Kavitha et al. 2012).Determination of alveolar bone loss and residual alveolar ridge resorption are often made by using panoramic radiographs. One of the best established methods to assess the mandibular cortical width is panoramic mandibular index, which was first introduced by Benson in 1991. (Duncea et al.2013).

In this regard, the present study has aimed to evaluate and compare salivary calcium and panoramic mandibular index, so as to identify the risk group of osteoporosis in postmenopausal women. 


\section{Methodology}

A case control study was performed in the Department of Oral Medicine, Oral Diagnosis and Radiology, Vishnu Dental College and Hospital, Bhimavaram, Andhra Pradesh, India.

A total of 60 females were included in the study. The procedure was explained to the patient and informed consent was obtained from individual patient. The study was approved by the institutional Ethical committee.

Patients with certain systemic diseases (Diabetes, hypertension. thyroid disorders, any developmental disorders of mandible, fractures or surgery of the mandible) were excluded from the study. The total sample of 60 females was categorized into two groups. Control group: Comprised of 30 healthy women (age range 25-40 years) having regular menstrual cycle.

Case group: Comprised of 30 healthy postmenopausal women (age range 41-60years) with natural occurrence of menopause of at least 12 months to 15 years of span.

\subsection{Collection of saliva}

The patients were comfortably seated in the Dental chair and unstimulated saliva was collected from individual patient under resting conditions. The saliva was collected for 10 minutes by spitting method in a graduated container from 9AM to 12PM to avoid diurnal variations. The collected saliva was then subjected to biochemical analysis for assessment of salivary calcium levels.

\subsection{Salivary calcium analysis}

The collected saliva was centrifuged at 3000RPM and supernatants were separated. To the obtained supernatants, reagents were added and concentration of calcium was calorimetrically analysed by using spectrophotometry. The findings were expressed in $\mathrm{mg} / \mathrm{dl}$.

\subsection{Radiographic analysis}

Orthopanoramic images were taken for each patient as an adjuvant for various diagnostic and treatment purposes. Panoramic image was obtained using PANOCEPH machine with Photostimulable phosphor plates. Linear measurements were used and specific calibrations were performed with the Digora software for windows 2.7.103.437 in order to calculate panoramic mandibular index.

\subsection{Calculation of Panoramic mandibular index}

A tangent was drawn across the lower margin of mandible followed by which the width of mandibular cortex at the mental area is measured as shown in figure.1. A perpendicular line was then drawn from the mental foramen upto the tangent and this distance was measured as shown in figure. 1

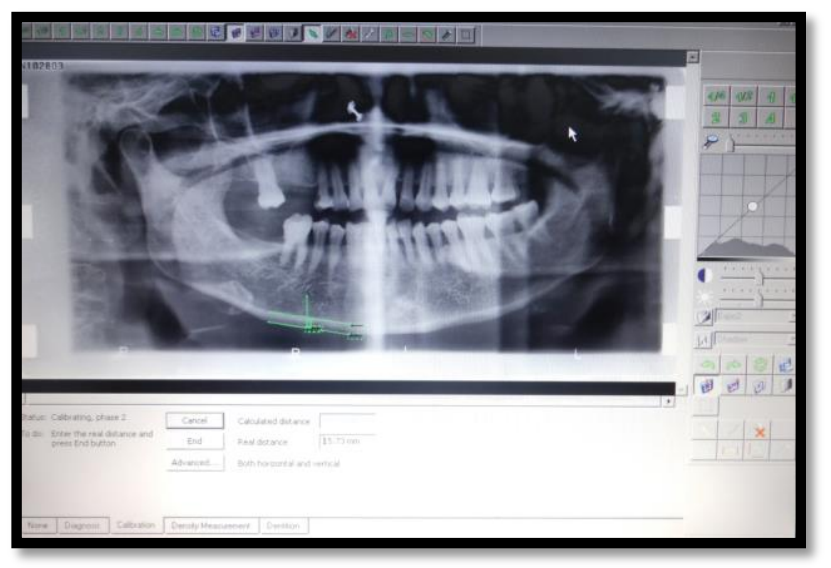

Fig. 1: Showing the Tools Used and Calibrations performed to Calculate Panoramic Mandibular Index
Panoramic mandibular index was further calculated as the ratio between the width of the lower mandibular cortex in the mental area and the distance between the lower margin of the mandible and the mental foramen as proposed by BENSON (Duncea et al.2013).

\section{Results}

A total of 60 women were enrolled in the study. The mean age among the case group was obtained as 53.43 years and among the control group was 32.5 years. The comparison of age among the case and control subjects is shown in Table.1.

Table 1: Comparison of Age among the Case and Control Group (P Value $=0.001)$

\begin{tabular}{lllll}
\hline GROUPS & $\mathrm{N}$ & Mean & $\begin{array}{l}\text { Std. Devia- } \\
\text { tion }\end{array}$ & P-VALUE \\
\hline CASE GROUP & 30 & 53.43 & 4.973 & $0.001^{*}$ \\
CONTROL GROUP & 30 & 32.56 & 5.787 & \\
\hline
\end{tabular}

In the current study Mann-Whitney U test was performed and the mean salivary calcium levels among the case group was obtained as $6.9 \mathrm{mg} / \mathrm{dl}$ and the control group was $4.8 \mathrm{mg} / \mathrm{dl}$ mean which depicts that salivary calcium among the case group was significantly higher than the control group $(\mathrm{p}$ value $=0.001)$ as shown in Table. 2

Table 2: The Mean Salivary Calcium Level Is Significantly Higher in Case Group $(\mathrm{P}$ Value $=0.001)$

\begin{tabular}{llcccc}
\hline & GROUPS & $\mathrm{N}$ & Mean & $\begin{array}{l}\text { Std. Devi- } \\
\text { ation }\end{array}$ & $\begin{array}{l}\text { P- } \\
\text { VALUE }\end{array}$ \\
\hline $\begin{array}{l}\text { SALIVARY } \\
\begin{array}{l}\text { CALCIUM } \\
(\mathrm{mg} / \mathrm{dl})\end{array}\end{array}$ & $\begin{array}{l}\text { CASE } \\
\text { GROUP }\end{array}$ & 30 & 6.93 & .89 & \\
\hline & CONTROL & 30 & 4.87 & .55 & $0.001 *$ \\
\hline
\end{tabular}

The mean PMI levels among the case and control groups were 0.3 and 0.43 respectively and illustrates that mean PMI values were significantly lower than the control group $(\mathrm{p}$ value $=0.001)$ as shown in Table. 3 .

Table 3: The Mean PMI Level Is Significantly Lower in Case Group when Compared with the Control Group (P Value $=0.001)$

\begin{tabular}{llllll}
\hline & GROUPS & $\mathrm{N}$ & MEAN & $\begin{array}{l}\text { Std. } \\
\text { Deviation }\end{array}$ & $\begin{array}{l}\text { P- } \\
\text { VALUE }\end{array}$ \\
\hline \multirow{3}{*}{ PMI } & CASE & 30 & 0.30 & .06 & \\
& $\begin{array}{l}\text { GROUP } \\
\text { CONTROL } \\
\text { GROUP }\end{array}$ & 30 & 0.43 & .054 & $0.001^{*}$ \\
\hline
\end{tabular}

Pertaining to the duration of the menopause, the correlation coefficient of salivary calcium and PMI were obtained as 0.86 and 0.705 and significant (2-tailed) test has ruled out that as the duration of menopause is increasing, the salivary calcium levels were increasing and PMI values were found to be decreasing and both were statistically significant $(\mathrm{p}$ value $=0.001)$ as shown in Table 4.

Table 4: Significant Increase in Salivary Calcium Levels and Significant Decrease in PMI Levels with Advancing Duration of Menopause (P Value $=0.001$ )

\begin{tabular}{llll}
\hline & & $\begin{array}{l}\text { SALIVARY } \\
\text { CALCIUM (mg/dl) }\end{array}$ & PMI \\
\hline \multirow{2}{*}{ MENOPAUSE } & Correlation & $0.867^{* *}$ & - \\
DURATION & Coefficient & $0.705^{* *}$ \\
& Sig. (2-tailed) & $0.001^{*}$ & $0.001^{*}$ \\
\hline
\end{tabular}

\section{Discussion}

Postmenopausal women are at a greater risk for osteoporosis which is attributed by low estrogen levels. Estrogen deficiency tends to cause both direct and indirect effects on the skeletal sys- 
tem (Wolfgang Sipos et al. 2009 )which thereby leads to condition called Osteoporosis and is often accompanied by increased resorption of bone(Siva Reddy et al. 2008).

Osteoporosis is one of the most common metabolic diseases associated with ageing and goes hand in hand with increasing duration of menopause.

In the research study conducted by Singh et al., he had described that estrogen deficiency in postmenopausal women leads to decreased absorption of calcium in intestine that implies a decreased serum calcium level, as a result of which PTH hormone level increases in serum which is accompanied by increase in the serum calcium. Nevertheless, in order to maintain calcium homeostasis, there is no significant increase in serum calcium; therefore there is a possibility of elevated calcium to be excreted through saliva or kidney. (Singh et al. 2013)

Previous studies have exemplified the mean age for the postmenopausal women as 58.78 years (PR Bodade et al 2013), 54.26years (Mudda, et.al 2010), 55.5years (Vishwanath, et al. 2011) the mean age of the post-menopausal women in this study was 53.43years which closely correlates with the previous studies.

Saliva is called ultra-filtrate of plasma (Kavitha et al. 2012) and several researches have implicated the diagnostic value of salivary constituents in screening various diseases. Calcium is an essential mineral in the bone as well as an important inorganic component in the saliva. Increased resorption of bone in postmenopausa women results in passive diffusion of calcium into the saliva via serum and as a consequence of which calcium levels are found to be increased in saliva.

In the current study, the mean value of the salivary calcium in postmenopausal women was obtained as $6.9 \mathrm{mg} / \mathrm{dl}$ which was above the cut-off point $(6.1 \mathrm{mg} / \mathrm{dl})$ as reported by Maryam Rabiei.et al. (2012). This result elicits that salivary calcium levels above $6.1 \mathrm{mg} / \mathrm{dl}$ can indicate the possible occurrence of osteoporosis.

A study conducted by R. Singh et al (2012), F Agha-Hosseini et al. (2011) has reported a significant difference in mean values of salivary calcium concentration in postmenopausal women which is in accordance with the present study.

In this study, salivary calcium levels are increasing significantly with the age in menopause women attributing to the fact that there is increased bone resorption and decreased bone density which is in accordance with the study conducted by (Sewon et al. 2004) wherein there was a significant increase in salivary calcium levels with low mineral density.

Clinicians had focused on various radiomorphometric indices to assess the mandibular cortical width and substantiate their role as a potential screening tool for osteoporosis (Haster et al. 2011). Previous studies have demonstrated that panoramic mandibular index had a greater efficacy in identifying the postmenopausal women with osteoporosis. (Yashoda et al. 2011)

Yashoda et al. (2011) has recommended a mean optimal cut off value of PMI as 0.25 for osteoporosis which is in close approximation with the present study which revealed a mean PMI of 0.3 .

The mean value of PMI in the present study was in close harmony with the study conducted by (Haster et al. 2011)., where the results had showed a mean PMI value of 0.27 among the elderly women, which infers that increased resorption of bone results in decreased bone mass which can be assessed by decrease in PMI values and thus it can be suggested that $\mathrm{PMI}<0.3$ can be used as a screening tool of osteoporosis.

Earlier study reported by (Bosky Gaur et al. 2013), demonstrated the PMI values ranging between 0.27 to 0.34 among postmenopausal women which strongly correlates with the present study where the latter one has its values ranging between 0.21-0.39 Khojastehpour et al. (2009) and Ramesh et al. (2011) has found a strong negative correlation between age and the mean PMI, which states that PMI was found to be decreasing with the advancing age as similar to this study.

Saliva, as an adjuvant to the panoramic radiographs is beneficial to the patient as well as the physician since these methods are noninvasive, painless and economical. As per the previous document- ed literature and to the best for our knowledge, this is probably the first study comparing the salivary calcium levels and the PMI so as to indentify the risk of occurrence of osteoporosis among postmenopausal women.

\section{Conclusion}

Pertaining to the results of the current study, both salivary calcium and panoramic mandibular index has played an equal important role in identifying the risk group of osteoporosis at an early stage and thus these parameters can be regarded as ancillary methods in early diagnosis of osteoporosis.

\section{References}

[1] Poornima Govindraju and Poornima Chandra (2014) Radiomorphometric Indices of the Mandible - An Indicator of Osteoporosis Journal of Clinical and Diagnostic Research. Mar, Vol-8(3): 195-198.

[2] Hastar E, Yilmaz H, Orhan H. Evaluation of Mental Index (2011) Mandibular Cortical Index and Panoramic Mandibular Index on Dental Panoramic Radiographs in the Elderly. European Journal Dentistry. 5:60-67.

[3] Siva Reddy et al., (2008) Oral Signs and Salivary Parameters as Indicators of Possible Osteoporosis and Osteopenia in Postmenopausal Women - A Study of 45 Subjects Brazilian Journal Oral Sciensec. 7(24):1502-1506.

[4] M. Rabiei, Masooleh IS, Leyli EK, Nikoukar LR. Salivary calcium concentration as a screening tool for postmenopausal osteoporosis. International Journal of Rheumatic Diseases 2012; 1-5.

[5] Kavitha MS, Asano A, Taguchi A, Kurita T, Sanada M.(2012) Diagnosis of osteoporosis from dental panoramic radiographs using the support vector machine method in a computer-aided system. BMC Medical Imaging; 12(1):1-11. http://dx.doi.org/10.1186/1471-2342-12-1.

[6] Duncea I, Pop A, Georgescu CE. (2013), the relationship between osteoporosis and the panoramic mandibular index. International Journal of the Bioflux Society; 5(1):14-18.

[7] Wolfgang Sipos (2009) Pathophysiology of osteoporosis. Wien Med Wochenschr 200: 159/9-10: 230-234.

[8] Singh B, Sheikh S, Pallagatti S, Kaur K, Sohi R. (2013), Oral dryness with salivary parathyroid hormone and salivary calcium. Contemporary Clinical Dentistry; 4 (4):488-492. http://dx.doi.org/10.4103/0976-237X.123051.

[9] Bodade PR, Mody NR. (2013)Panoramic Radiography for Screening Postmenopausal Osteoporosis in India: A Pilot Study. OHDM; 12 (2):6572.

[10]Mudda JA, Bajaj M, Patil VA. (2010) A Radiographic comparison of mandibular bone quality in pre- and post-menopausal women in Indian population. J Indian Soc Periodontol.; 14(2): 121-125. http://dx.doi.org/10.4103/0972-124X.70833.

[11] Vishwanath SB, Kumar V, Kumar S, Shashikumar P, Shashikumar Y, Patel PV. (2011) periodontal status and bone mineral density. Indian Journal of Dental Research; 22(2):270-276.

[12]F. Agha Hosseini, Dizgah IM, and Moosavi MS. (2012) Relationship of serum and saliva calcium, phosphorus and alkaline phosphatase with dry mouth feeling in menopause, Gerodontology; 29: 1092-1097. http://dx.doi.org/10.1111/j.1741-2358.2012.00619.x.

[13] R Singh et al. (2012), Correlation of serum oestrogen with salivary calcium in postmenopausal women with and without oral dryness feeling, Gerodontology; 29: 125-129. http://dx.doi.org/10.1111/j.1741-2358.2011.00580.x.

[14]F Agha-Hosseini, Dizgah IM, Moghaddam PP, Akrad ZT (2007). Stimulated whole salivary flow rate and composition in menopausal women with oral dryness feeling. Oral Diseases; 1:320-323. http://dx.doi.org/10.1111/j.1601-0825.2006.01288.x.

[15] Sewon L, Lainea M, Karjalainenb S, Doroguinskaiaa A, Veromaa ML. (2004) Salivary calcium reflects skeletal bone density of heavy smokers. $\begin{array}{llll}\text { Archives of Oral Biology 49, 355-358. } & \text {. }\end{array}$ http://dx.doi.org/10.1016/j.archoralbio.2003.11.004.

[16] Yashoda Devi BK, Rakesh N, Ravleen N. (2011). Diagnostic efficacy of panoramic mandibular index to identify postmenopausal women with low bone mineral densities. Journal of Clinical and Experimental Dentistry; 3(5):456-61. http://dx.doi.org/10.4317/jced.3.e456.

[17] Gaur B, Choudary A, Wanjari PV, Sunil MK, Basvaraj P. (2013). Evaluation of panoramic radiographs as a screening tool of osteoporosis in Postmenopausal women: A Cross sectional study. Journal of Clinical and Diagnostic Research; 7(9):2051-2055.

[18] Khojastehpour L, Shahidi SH, Barghan S, Aflaki EL (2009);. Efficacy of Panoramic Mandibular Index in Diagnosing Osteoporosis in Women. Journal of Dentistry6 (1):11-15

[19]Ramesh A, Mahajan K, Thomas B, Shenoy N, Bhandary R (2011). Alveolar bone mass in pre- and postmenopausal women with serum calcium as a marker: A comparative study. Indian J Dent Res; 22:878. http://dx.doi.org/10.4103/0970-9290.94667. 